\title{
Negative Skin Friction on Piles Based on Finite Strain Consolidation Theory and the Nonlinear Load Transfer Method
}

\author{
Hyeong Joo Kim* and Jose Leo C. Mission**
}

Erratum to:

KSCE Journal of Civil Engineering (2009) 13(2):107-115

DOI 10.1007/s12205-009-0107-7

The original version of this article unfortunately contained a mistake.

Errata:

1. Eq. (6):

$\zeta=1-\frac{1}{\bar{\varepsilon}}=\exp (\varepsilon) \rightarrow \zeta=\frac{1}{1-\bar{\varepsilon}}=\exp (\varepsilon)$
2. Eq. (8):

$$
\zeta(0, t)=1-\frac{1}{\bar{\varepsilon}}=\exp (\varepsilon) \rightarrow \zeta(0, t)=\frac{1}{1-\bar{\varepsilon}}=\exp (\varepsilon),
$$

for a free draining (permeable) upper boundary

3. Eq. (9):

$$
\zeta\left(H_{o}, t\right)=1-\frac{1}{\bar{\varepsilon}}=\exp (\varepsilon) \rightarrow \zeta\left(H_{o}, t\right)=\frac{1}{1-\bar{\varepsilon}}=\exp (\varepsilon),
$$

for a free draining (permeable) lower boundary

*Member, Professor, School of Civil and Environmental Engineering, Kunsan National University, Kunsan 573-70, Korea (Corresponding Author, E-mail: kimhj@kunsan.ac.kr)

**Graduate Student, School of Civil and Environmental Engineering, Kunsan National University, Kunsan 573-70, Korea (E-mail: joseleo_mission@ kunsan.ac.kr) 